\title{
Strength and Coordination Training Are Both Effective in Reducing the Postural Tremor Amplitude of Older Adults
}

\author{
Justin W.L. Keogh, Steve Morrison, and Rod Barrett
}

The current study investigated the effect of 2 different types of unilateral resistance training on the postural tremor output of 19 neurologically healthy men age $70-80 \mathrm{yr}$. The strength- $(n=7)$ and coordination-training $(n=7)$ groups trained twice a week for $6 \mathrm{wk}$, performing dumbbell biceps curls, wrist flexions, and wrist extensions, while the control group $(n=5)$ maintained their normal activities. Changes in index-finger tremor (RMS amplitude, peak, and proportional power) and upper limb muscle coactivation were assessed during 4 postural conditions that were performed separately with the trained and untrained limbs. The 2 training groups experienced significantly greater reductions in mean RMS tremor amplitude, peak, and proportional tremor power $8-12 \mathrm{~Hz}$ and upper limb muscle coactivation, as well as greater increases in strength, than the control group. These results further demonstrate the benefits of resistance training for improving function in older adults.

Keywords: aging, exercise therapy, upper limb, weight training

A decline in upper limb dexterity is commonly associated with the normal aging process (Carmeli, Patish, \& Coleman, 2003; Hackel, Wolfe, Bang, \& Canfield, 1992; Ranganathan, Siemionow, Saghal, \& Yue, 2001). A potential contributing factor to this loss of upper limb function in older adults is the age-related increase in postural tremor amplitude (Birmingham, Wharrad, \& Williams, 1985; Loscher \& Gallasch, 1993; Morrison, Mills, \& Barrett, 2006). The increased tremor amplitude for older adults is most pronounced between 8 and $12 \mathrm{~Hz}$ (Morrison et al.) and may be related to their greater motor-unit firing-rate variability (Laidlaw, Bilodeau, \& Enoka, 2000) and upper limb muscle coactivation (Laursen, Jensen, \& Ratkevicius, 2001; Spiegel, Stratton, Burke, Glendinning, \& Enoka, 1996).

Although the index-finger postural tremor signal is composed of several frequency peaks, the 8 - to $12-\mathrm{Hz}$ peak is generally the largest in magnitude for unsupported postural pointing tasks, accounting for $\sim 60 \%$ of total tremor power (Keogh, Morrison, \& Barrett, 2004; Morrison et al., 2006). The 8- to 12-Hz tremor

Keogh is with the Centre for Physical Activity and Nutrition Research, Auckland University of Technology, Auckland, New Zealand. Morrison is with the School of Physical Therapy, Old Dominion University, Norfolk, VA. Barrett is with the School of Physiotherapy and Exercise Science, Griffith University, Gold Coast, Australia. 
peak is referred to as the neurogenic component because it is thought to reflect the output of central oscillators that modulate alpha-motoneuron activity, as well as the feedback from stretch reflexes (McAuley \& Marsden, 2000).

The age-related increases in the tremor amplitude of some older adults may represent a general loss of neuromuscular function or a decline in muscle strength (Sosnoff \& Newell, 2006). This age-related loss of strength could reflect changes in a number of neural processes such as increased coactivation and altered motor-unit firing, as well as several morphological changes (Enoka et al., 2003). According to Brunner et al. (2007), the primary morphological factors related to older adults' reduced strength would be the decrease in their proportion, number, and size of fast-twitch muscle fibers or motor units. Accordingly, interventions that improve older adults' upper limb neuromuscular function and strength may reduce the overall neuromuscular decline associated with aging and the resulting tremor amplitude.

Given that resistance training can significantly improve strength (FiataroneSingh, 2002; Hakkinen et al., 1998) and muscle hypertrophy (Kosek, Kim, Petrella, Cross, \& Bamman, 2006), as well as reduce motor-unit firing-rate variability (Kornatz, Christou, \& Enoka, 2005) and coactivation (Carolan \& Cafarelli, 1992; Hakkinen et al.), resistance training may be effective in reducing the tremor amplitude of neurologically healthy older adults. Ranganathan, Siemionow, Saugen, Liu, and Yue (2001) reported a significant increase in hand steadiness (an indirect measure of tremor amplitude) of the trained limb during a dexterous finger-pinching task in neurologically healthy older adults after an 8-week coordination-training program. However, these changes in hand steadiness were only examined in the trained limb during the performance of a task that shared a number of similarities to that performed in training (Ranganathan, Siemionow, Saugen, et al.). Thus, it remains unclear whether general (nonspecific) unilateral resistance training can reduce the tremor amplitude of older adults in dexterous tasks that share little similarity to those practiced in training. Furthermore, it is unclear whether such effects can be observed in the untrained limb, as well as the trained limb, a process known as cross-education (Lee \& Carroll, 2007). If it can be demonstrated that resistance training can significantly reduce older adults' tremor amplitude in tasks not practiced in training or if such effects could be observed in the untrained limb, the potential applications of resistance training in this population would be increased.

The purpose of the current study was to determine whether two forms of resistance training, one very general (strength training) and the other semispecific (coordination training) in nature, could reduce the tremor amplitude of the trained and untrained limbs of neurologically normal older adults. We hypothesized that both forms of resistance training would significantly increase upper limb strength and decrease postural tremor amplitude. Consistent with the principle of specificity, we hypothesized that the reduction in tremor amplitude would be more pronounced in the coordination- than strength-training group. Because the benefits of training were expected to be reflected by primarily neural changes, any reductions in indexfinger-tremor amplitude were expected to be confined to the 8- to $12-\mathrm{Hz}$ range. In addition, a general decline in upper limb muscle coactivation was expected to result from training. 


\section{Methods}

\section{Participants}

Twenty-one participants were recruited for this study, with 7 allocated to each of the strength- and coordination-training and control groups. Power analyses indicated that 21 participants would give at least $80 \%$ power to detect significant trainingrelated changes in strength with a risk of Type I error of $<5 \%$. Unfortunately, before conducting this study there were no data that could be used to perform a power analysis for our primary outcome measure, tremor amplitude. On this basis, our study could be considered a pilot study, with the results informing future studies in this area.

Entry criteria for the current study included being male, age 70-80 years, physically active and of apparent good health, and community dwelling and not having been involved in regular resistance training during the last year. All participants needed to be free from ocular or systemic disease, having no recent or recurrent history of musculoskeletal injury, no neurological conditions, no history of diabetes or vertigo, no use of an aid while walking, no difficulty standing upright, and no visible tremor or uncorrected visual deficits. This meant that the participants were all competent in performing basic and instrumental activities of daily living.

The first 7 participants who gave informed consent were allocated to the strength-training group, the next 7 to the coordination-training group, and the last 7 to the control group. Although this approach is inferior to randomly allocating participants to the three groups via a randomized controlled trial model, such an approach has been used previously in a comparable study (e.g., Bilodeau, Keen, Sweeney, Shields, \& Enoka, 2000). In the control group, 2 participants dropped out before the posttraining testing session. Consequently, only 5 control participants completed both testing sessions. Attendance at the training sessions was very high (99.4\%) for the two training groups, with only 1 participant missing a training session. The university human research ethics committee granted ethical approval for this study. The baseline demographic characteristics of the three groups are presented in Table 1.

\section{Experimental Design}

The current study was conducted to assess the ability of two unilateral dumbbellbased resistance-training programs to reduce the postural tremor output of older

Table 1 Baseline Demographic Characteristics of the Three Groups, $M \pm S D$

\begin{tabular}{lccc}
\hline & $\begin{array}{c}\text { Strength-training } \\
\text { group }(\boldsymbol{n}=\mathbf{7})\end{array}$ & $\begin{array}{c}\text { Coordination-training } \\
\text { group }(\boldsymbol{n}=\mathbf{7})\end{array}$ & $\begin{array}{c}\text { Control group } \\
(\boldsymbol{n}=\mathbf{5})\end{array}$ \\
\hline Age (years) & $75 \pm 2$ & $75 \pm 3$ & $76 \pm 3$ \\
Height $(\mathrm{cm})$ & $171 \pm 6$ & $174 \pm 7$ & $179 \pm 5$ \\
Mass $(\mathrm{kg})$ & $72 \pm 7$ & $86 \pm 21$ & $87 \pm 12$ \\
Body-mass index $\left(\mathrm{kg} / \mathrm{m}^{2}\right)$ & $25 \pm 3$ & $28 \pm 6$ & $27 \pm 4$ \\
\hline
\end{tabular}


adults. Changes in tremor output and forearm muscle activity were assessed in four pointing tasks of varying levels of complexity. We did this because previous research indicated that changes in the task goals have the potential to alter the tremor output and coordinative patterns used (Keogh et al., 2004; Morrison \& Keogh, 2001). Each of these tasks was performed unilaterally with the trained and untrained limb for a total of eight conditions. Six 30-s trials of each of the eight postural pointing tasks were performed in randomized block order in both the preand posttraining testing sessions.

The four pointing conditions performed by each participant were pointing with no external target (no target, NT), pointing the laser at a blank (featureless) target area (BT), pointing at a concentric-circle target situated close to the participant (close target, CT), and pointing at a concentric-circle target farther from the participant (far target, FT). For the NT condition, the laser pointer was switched off and the participants focused on minimizing the motion of the index finger. All other conditions (BT, CT, and FT) were performed with the laser pointer activated. The goal of the BT condition was to minimize the oscillation of the laser pointer emission on a featureless target located $5.50 \mathrm{~m}$ from the participant. For the CT and FT conditions, the aim was to keep the laser pointer emission within the smallest concentric circle (2-cm radius) of the target. The distances between the participant and target were $2.75 \mathrm{~m}$ and $5.50 \mathrm{~m}$, respectively, for the CT and FT conditions.

\section{Resistance-Training Procedures}

The strength- and coordination-training groups performed unilateral dumbbell training twice per week for 6 weeks. The arm selected for training was randomly determined on the basis of each participant's self-reported hand preference. Participants warmed up for each session by performing 5 min of arm cranking at a workload of $30 \mathrm{~W}$ on a modified Monark cycle ergometer (Monark Exercise, Varberg, Sweden). Four sets of each of three exercises (biceps curls, wrist flexions, and wrist extensions) were then performed by each group. The first set of each exercise was a specific warm-up involving $40-50 \%$ of the five-repetition-maximum (5RM) load. Thus, both groups performed exactly the same warm-up procedures, with the only difference in their training sessions being the manner in which the three training sets of each exercise was performed. Two to three minutes rest were given between sets. The control group performed no training and maintained their normal activities during the 6-week training period.

The strength-training group performed each exercise with loads that could only be lifted for 8-10 repetitions per set (Fiatarone-Singh, 2002). To make training progressively more difficult, once four sets of 10 repetitions could be completed the training load for that particular exercise was increased by $1 \mathrm{~kg}$ for the next session.

The goal of the coordination-training tasks was for the participants to match as closely as possible the angular motion of their limb segments to that of a predetermined, quasi-random joint angular displacement-time curve (target trajectory). This task goal was made explicit to the participants before each training session. This form of training was performed because the practice of similar force-matching tasks has been shown to significantly improve motor-unit control properties (Knight \& Kamen, 2004; Patten \& Kamen, 2000). Joint angular displacements were recorded 
with a custom-built rotary potentiometer at a rate of $50 \mathrm{~Hz}$. The potentiometer was aligned with the center of rotation of the selected joint and securely strapped to the proximal and distal limb segments.

The target trajectories for each exercise were developed through pilot testing with the intent of creating challenging tasks in which considerable improvement in task performance (accuracy) could occur with 6 weeks of practice. Each of the exercise's target trajectories consisted of 13 peaks (partial repetitions), with the mean velocity of the concentric and eccentric phases being $~ 50 \%$ s. This velocity was used because pilot testing demonstrated that it was appropriate for the participant group and because it was comparable to the mean velocity used by the strength-training participants in their training sessions. Figure 1 provides an example of an angular displacement-time profile for a representative participant performing a biceps curl in his first training session. The target trajectory and the participant's actual joint angular displacement were displayed in real time on a 17-in. ( 43-cm) computer monitor positioned $\sim 80 \mathrm{~cm}$ from the participant. To ensure sufficient visual resolution, the target and the participant's actual angular displacements covered the majority of the computer screen and were continually updated so that $\sim 8 \mathrm{~s}$ of data were seen on the screen at any one time.

Before starting the first training session, each participant in the coordinationtraining group was permitted a short period of practice to familiarize himself with the coordination-training tasks. This practice was only permitted during the first session. For the first 2 weeks (Phase 1) of training, the coordination group used loads

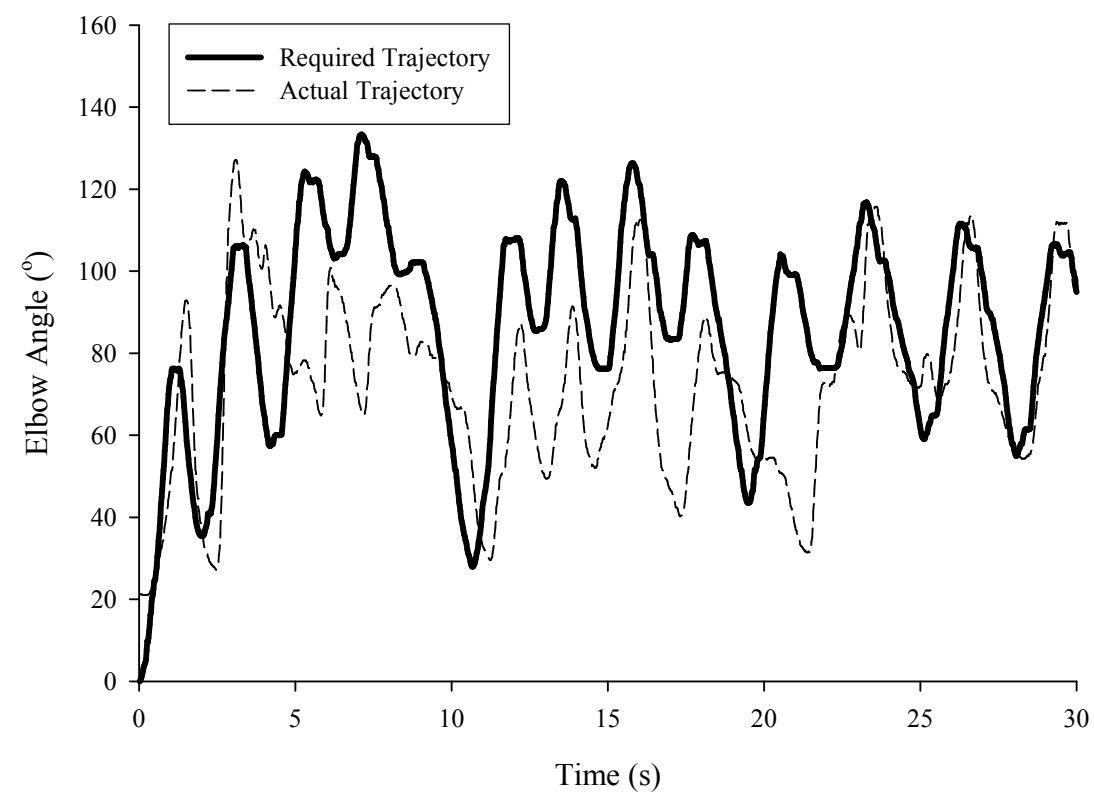

Figure 1 - Actual versus required angular trajectory of the elbow joint during the performance of a biceps curl by a representative participant in the coordination-training group during their first training session. Elbow extension is represented by $0^{\circ}$. 
that were $\sim 30 \%$ of their 5RM. These loads were increased by $1 \mathrm{~kg}$ every 2 weeks (Phases 2 and 3 ) to make training progressively more difficult. Because pilot testing had indicated that the strength-training group required $\sim 30$ s to complete each of their 8- to 10-repetition sets, the sets performed by the coordination-training group were also $30 \mathrm{~s}$ in duration. This was to control for the total contractile (exercise) time between the two training groups (Moss, Refsnes, Abildgaard, Nicolaysen, \& Jensen, 1997).

\section{Assessment Procedures}

PosturalTremor. All postural pointing conditions were performed standing, with the selected arm flexed at the shoulder to $90^{\circ}$, the elbow fully extended, forearm pronated, and wrist held in a neutral position (Morrison \& Keogh, 2001). A laser pointer (length $14 \mathrm{~cm}$, mass $39 \mathrm{~g}$ ) was securely strapped to the palmar surface of the extended index finger for all conditions (Keogh et al., 2004). Tremor from the index finger, hand, forearm, and upper arm were recorded simultaneously by four lightweight (2.5-g) uniaxial Coulbourn T45-10 accelerometers (Coulbourn, Allentown, PA, USA) during all postural pointing conditions. Accelerometer signals were sampled at $100 \mathrm{~Hz}$, amplified through a Coulbourn transducer coupler (excitation voltage $5 \mathrm{~V}$, gain 1,000), and filtered using a second-order Butterworth low-pass filter (cutoff frequency $50 \mathrm{~Hz}$ ).

Electromyographic (EMG) activity of the extensor digitorum and flexor digitorum superficialis muscles was recorded to reflect the general activity of the wrist and finger extensors and flexors, respectively, using bipolar Ag/AgCl EMG electrodes (Medeleq, Nerang, Australia). In accordance with the recommendations of DeLuca (1997), these electrodes were positioned on the midline of the belly of each muscle parallel to the direction of the underlying muscle fibers with an interelectrode distance of $2 \mathrm{~cm}$. The position of these electrodes with respect to a number of selected bony landmarks was recorded in the pretest to ensure reliable electrode placement in the posttest. All EMG signals were sampled at 1,000 Hz, amplified using Coulbourn isolated bioamplifiers (V75-02), and filtered with a second-order Butterworth band-pass filter between 1 and $400 \mathrm{~Hz}$.

After application of the EMG electrodes, the participants performed a series of finger-extensor and -flexor maximal voluntary contractions (MVCs). The MVCs were performed in a seated position with the elbow of the selected arm directly under the shoulder and bent to $\sim 90^{\circ}$ with the forearm and wrist supported. After performing a number of submaximal isometric finger-extension and -flexion contractions, each participant performed three MVC trials (for both actions) with each limb. Each MVC was held for $\sim 4$ s, with 60 s rest between contractions.

Upper Limb Strength. Upper limb strength, as measured using the unilateral dumbbell biceps-curl, wrist-flexion, and wrist-extension exercises, was determined using a 5RM protocol similar to that of Hrysomallis and Kidgell (2001). All 5RM assessments were performed seated, with the upper body braced on the backrest of a sturdy chair. After 5 min of arm cranking on the modified Monark cycle ergometer, participants completed two progressively heavier warm-up sets of biceps curls, comprising five repetitions each set. The heaviest weight lifted for five repetitions was designated the 5RM. After determining the 5RM biceps-curl load for the preferred and nonpreferred limbs, this process was repeated for the 
wrist-flexion and -extension exercises. Excluding the two warm-up sets, the 5RM for each exercise was usually obtained in two or three attempts.

\section{Data Analysis}

The amplitude of the index-finger-tremor and EMG signals was obtained by calculating the root mean square (RMS) using a bin size of $100 \mathrm{~ms}$. The RMS EMG values were normalized to a percentage of the maximum RMS EMG amplitude obtained from the relevant MVC. The mean normalized extensor digitorum and flexor digitorum superficialis EMG amplitude values were then used to give an estimate of the degree of upper limb muscle coactivation via the calculation of the coactivation index (CI; Hortobagyi \& DeVita, 2000). The CI was defined as $\mathrm{FDS}_{\text {normalized }} / \mathrm{ED}_{\text {normalized }} \times 100$, where $\mathrm{ED}=$ extensor digitorum and $\mathrm{FDS}=$ flexor digitorum superficialis.

Because separate tremor peaks in the index-finger power spectrum may be observed at 2-4 Hz, 8-12 Hz, and 18-24 Hz, power spectral analysis was conducted within each of these three bandwidths. Within these separate bandwidths, peak power and the proportion of total power (proportional power) were calculated. All tremor- and EMG-data analyses were performed using custom-written MATLAB software (release 12 version 6.0, MathWorks, Natick, MA, USA).

The coordination-training group's performance was also quantified by calculating the RMS error. The RMS error was measured in degrees and gave an indication of the average absolute deviation between joint angles and the target trajectory.

\section{Statistical Analysis}

ANOVA was used to determine whether any significant between-groups differences occurred at baseline. The control group changed significantly over time (pre- vs. posttest), and the coordination-training group significantly reduced their RMS error in the coordination-training exercises.

Several significant differences were observed between the strength-training and control groups at baseline, so repeated-measures ANCOVA involving all three groups' data was used as the primary statistical test. All ANCOVAs were performed in SAS using the Proc-Mixed function. The "estimate" function was used to perform the post hoc comparisons, with the Tukey-Kramer post hoc test used to account for between-groups differences in sample size. Separate ANCOVAs were performed to examine the effect of two between factors (strength and coordination training) and up to two within factors (limb and condition) on each dependent variable. The condition effect was determined by comparing the tremor and CI change scores for the NT with the BT, CT, and FT conditions. We did this because previous studies have indicated that the augmented visual feedback provided by the activated laser pointer may result in increased tremor amplitude in unsupported postural pointing tasks (Keogh et al., 2004; Morrison \& Keogh, 2001). The between-groups difference (strength or coordination training versus control group) for the change (posttest minus pretest) scores was used as the effect statistic in all ANCOVAs. The pretest score for each variable was used as the covariate, because the pretest value for a particular variable is almost always the strongest predictor of its posttest score (A Collaborative Outcomes Research Network, 2007). All ANCOVAs used unilateral data for the strength- and coordination-training groups 
and bilateral (mean of the preferred and nonpreferred limb) data for the control group because they did not perform any training. All variables entered into the ANCOVAs were log transformed before analysis to reduce nonuniformity of the data (Hopkins, 2002; Keogh, Morrison, \& Barrett, 2007).

Cohen's effect size (ES) was calculated to determine the magnitude of the effect of strength and coordination training on the dependent variables (Cohen, 1988). According to Cohen, ESs $<.20,<.50,<.80$, and $>.80$ represent trivial, small, moderate, and large effects, respectively. The ES change score data are reported as $M \pm 95 \%$ confidence limit. All analyses were performed using SAS statistical software (SAS Institute, Lance Cove, Australia) with significance set at $p<.01$ to account for the relatively large number of statistical tests performed and the possible limitations of the ANCOVA models.

\section{Results}

Results of the baseline between-groups ANOVA revealed that all the significant between-groups effects were between the strength-training and control group. These analyses revealed that the strength-training group had significantly less tremor amplitude, $F(1,78)=13.70, p<.001$; wrist flexion, $F(1,34)=15.43, p<$ .001 ; and wrist extension, $F(1,34)=8.49, p<.001$, strength than the control group. No significant differences were observed for the control group over time (pre- vs. posttest) for any of the tremor or CI variables assessed in the postural pointing tasks, $F(1,78)=0.01-0.28, p=.618-.938$, or strength tests, $F(1,18)=0.06-2.25$, $p=.151-.809$. Similarly, the change scores for the strength- and coordinationtraining groups did not differ significantly on any postural pointing tremor or CI, $F(11,72)=0.51-2.22, p=.022-0.890, E S=-.34 \pm .31$ to $.06 \pm .28$, or strength, $F(11,30)=1.18-2.74, p=.014-.340, E S=-.47 \pm$ to $1.61 \pm 1.29$, variable.

\section{Strength and Coordination Training}

The strength-training group had a significantly greater increase in the trained limb's wrist-flexion strength, $F(11,30)=6.55, p<.001, E S=2.69 \pm 1.27$, and a nonsignificant although larger $(E S>.80)$ increase in the trained limb's biceps curl, $F(11,30)=$ $2.24, p=.039, E S=1.23 \pm 1.19$, and wrist extension, $F(11,30)=2.20, p=.043, E S$ $=0.92 \pm 0.90$, strength than the control group. The coordination-training group had a significantly greater increase in the trained limb's biceps-curl strength, $F(11,30)$ $=3.02, p=.008, E S=1.67 \pm 1.19$, and a nonsignificantly larger $(E S>.80)$ increase in the trained limb's wrist-flexion strength, $F(11,30)=2.40, p=.028, E S=1.39 \pm$ 1.23 , than the control group. Although there was a trend for increased strength in the untrained limb, this only reached statistical significance in the strength-training group for the wrist-flexion exercise, $F(11,30)=6.14, p<.001, E S=2.47 \pm 1.28$. The pre- and posttraining 5RM scores for all groups are presented in Figure 2.

Changes in the coordination-training group's performance were quantified by the RMS error. Although no significant changes in this measure were observed across the three exercises, the coordination-training group tended to reduce their RMS targeting error across the three 2 -week training phases, $F(2,60)=1.87, p=$ .162 , and across the four sessions within each of the 2 -week-long training phases, $F(3,80)=0.79, p=.505$. These changes are shown in Figure 3 . 

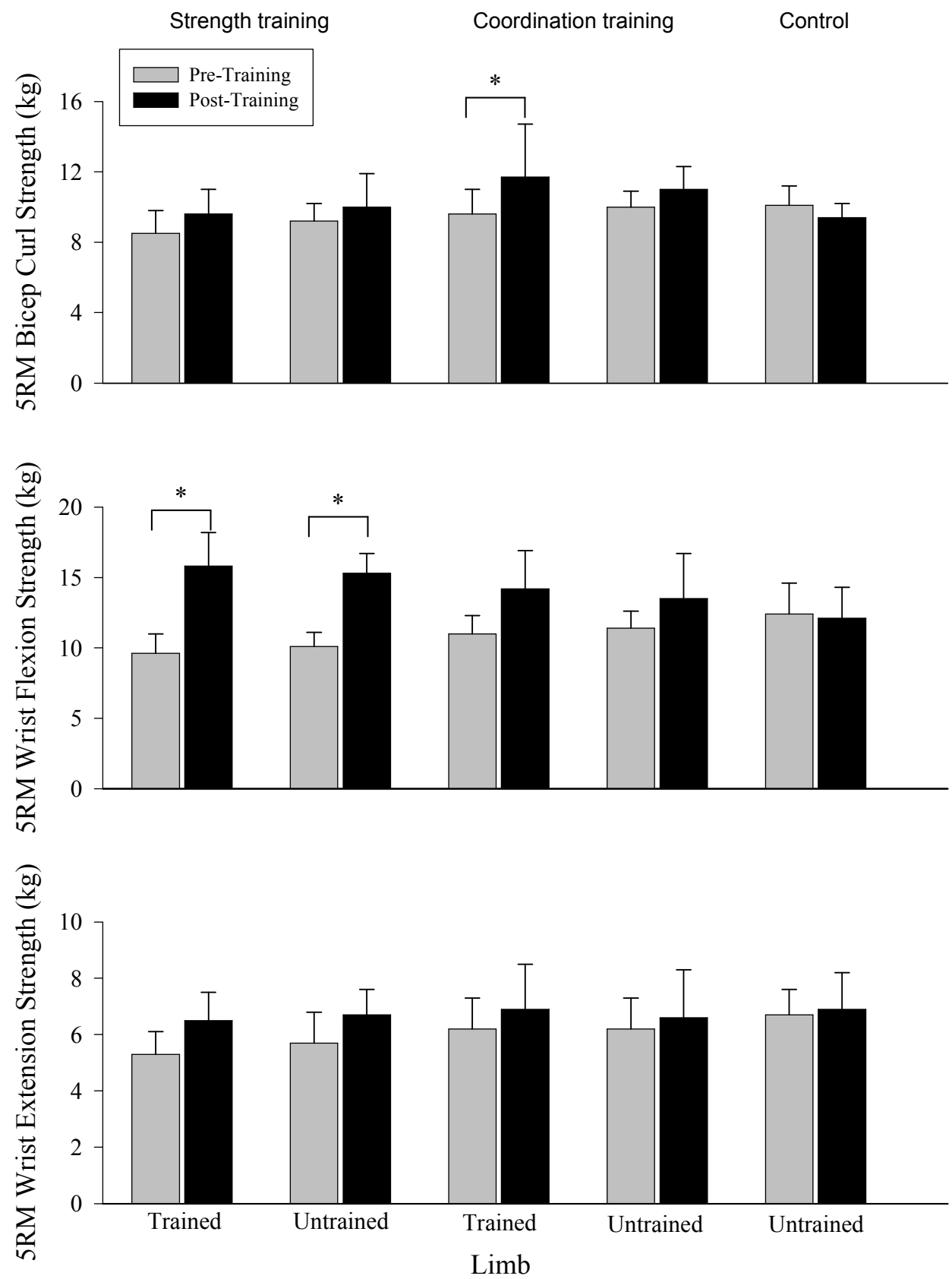

Figure 2 - Pre- and posttraining five-repetition-maximum (5RM) scores for the strength- and coordination-training and control groups, $M \pm S D$. * Significantly greater change for the training versus control group. 

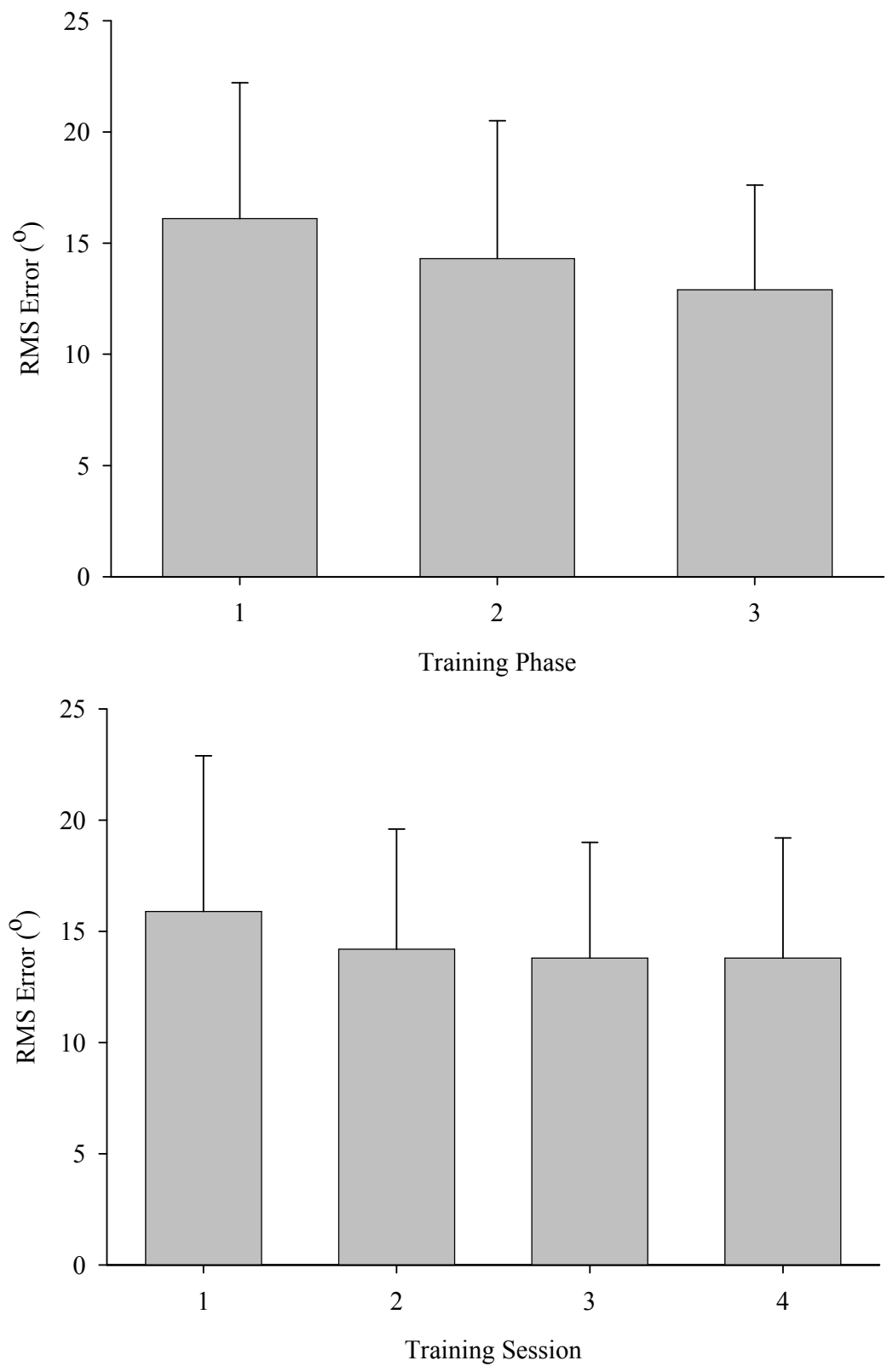

Figure 3 - Changes in the root-mean-square (RMS) error for the coordination-training group across and within each of the three 2 -week (four sessions) training phases. The data are collapsed across all three exercises because the training-related changes appeared similar across all exercises. 
Strength training
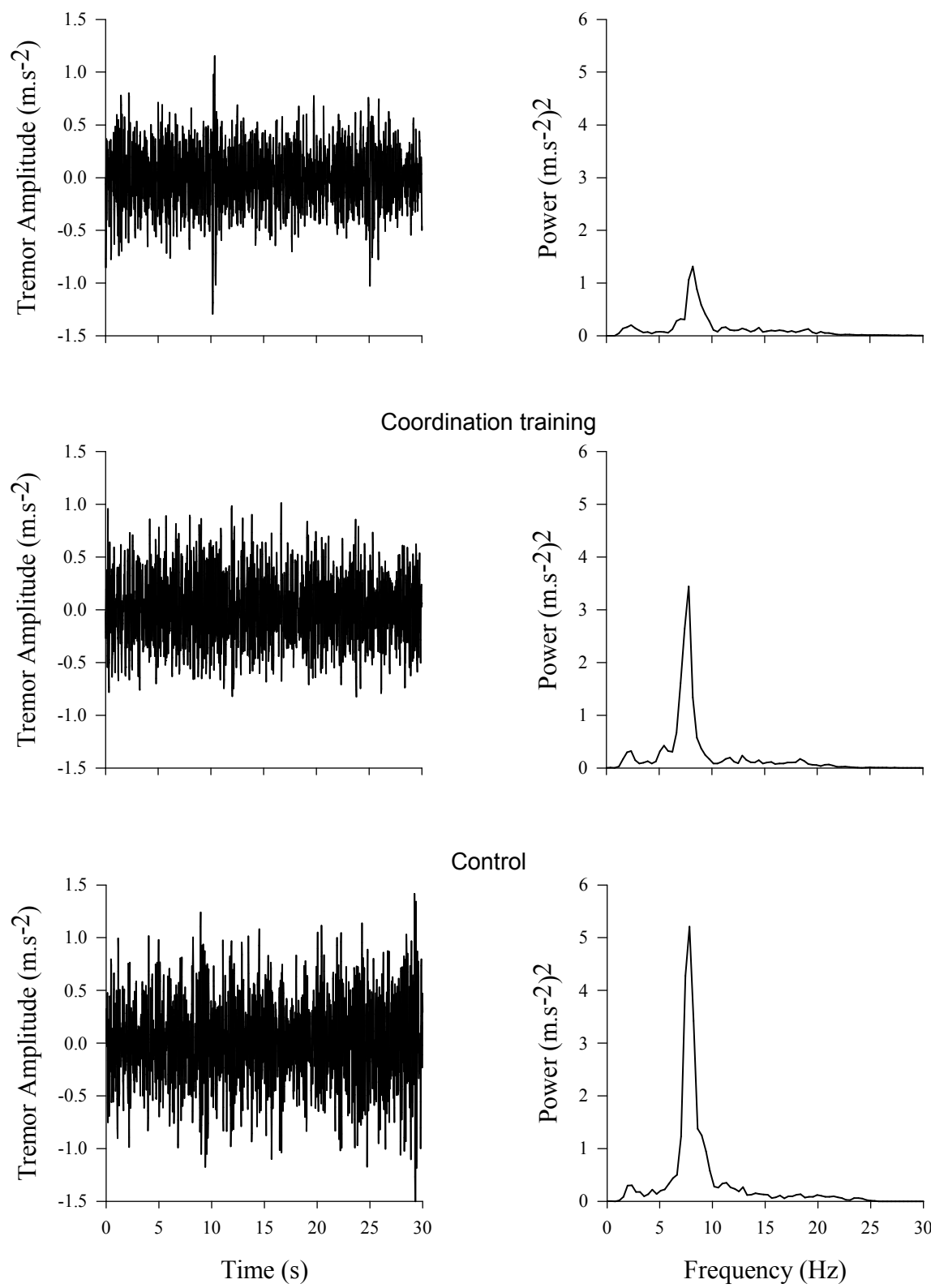

Control

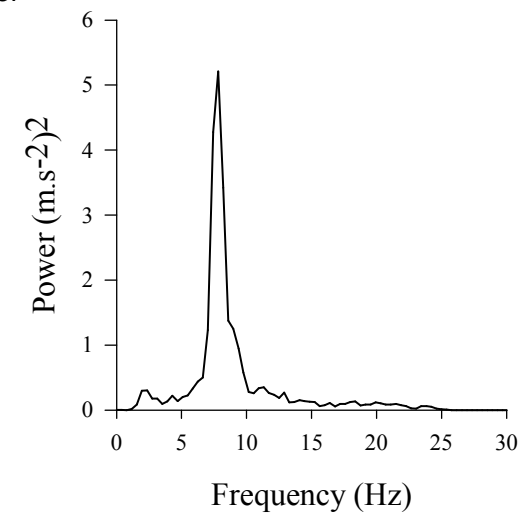

Figure 4 - Representative pretraining (baseline) index-finger-tremor time-series (left column) and frequency-domain (right column) data for the strength- and coordinationtraining and control groups. 


\section{Tremor Amplitude and Power Spectrum}

Representative time- and frequency-domain index-finger-tremor data for the strength- and coordination-training and control groups are presented in Figure 4.

The strength-training group, $F(11,72)=4.83, p<.001, E S=-.61 \pm .24$, and coordination-training group, $F(11,72)=3.81, p<.001, E S=-.44 \pm .22$, experienced significantly greater reductions in the trained limb's index-finger-tremor amplitude than the control group. Although some reductions in tremor amplitude were observed in the untrained limb, this reached statistical significance only for the coordination group, $F(11,72)=2.68, p=.006, E S=-.34 \pm .24$. Possible groupby-condition effects on the training-related change were assessed by comparing the between-groups change for the NT with the BT, CT, and FT conditions. No significant effects were observed for these comparisons in either training group, indicating that the training-related reductions in tremor amplitude were comparable across all four conditions. Accordingly, only the group-by-limb effects are presented throughout the article.

No significant differences were observed for the changes in the $2-$ to $4-\mathrm{Hz}$ and 18 - to $24-\mathrm{Hz}$ tremor power-spectrum variables (peak and proportional power) between the training and control groups. Peak 8- to $12-\mathrm{Hz}$ tremor power was significantly reduced for the strength-training group trained limb, $F(11,72)=4.64, p$ $<.001, E S=-.78 \pm .32$, and coordination-training group trained limb, $F(11,72)=$ $2.75, p=.005, E S=-.48 \pm .32$, and untrained limb, $F(11,72)=2.68, p=.006, E S$ $=-.38 \pm .27$, compared with the control group. In contrast, only the trained limb of the strength-training group had a significantly greater reduction in proportional 8 - to $12-\mathrm{Hz}$ power than the control group, $F(11,72)=2.75, p=.005, E S=-.48 \pm$ .32. The pre- and posttraining tremor amplitude, 8 - to $12-\mathrm{Hz}$ peak, and 8 - to $12-\mathrm{Hz}$ proportional tremor power scores for all groups are presented in Figure 5.

\section{Cl}

The reductions in the trained limb's CI for the strength-training group, $F(11,72)=$ $2.83, p=.004, E S=-.66 \pm .44$, and coordination-training group, $F(11,72)=3.34$, $p=.001, E S=-.53 \pm .31$, were significantly greater than for the control group. The pre- and posttraining CI scores for all groups are presented in Figure 6.

\section{Discussion}

Our primary hypothesis that two forms of resistance training (one general and the other semispecific) would significantly reduce the postural tremor amplitude of older adults was confirmed, with significant reductions in postural tremor amplitude observed for the trained limb of both training groups. This is an important finding because the aging process has been associated with increases in tremor amplitude (Birmingham et al., 1985; Loscher \& Gallasch, 1993; Morrison et al., 2006) and reduced upper limb dexterity (Carmeli et al., 2003; Hackel et al., 1992; Ranganathan, Siemionow, Saghal, and Yue, 2001). There was no significant difference in the tremor-amplitude change scores for the two resistancetraining groups, with the reductions in tremor amplitude being of a moderate and small effect size for the strength- and coordination-training groups, respectively. 

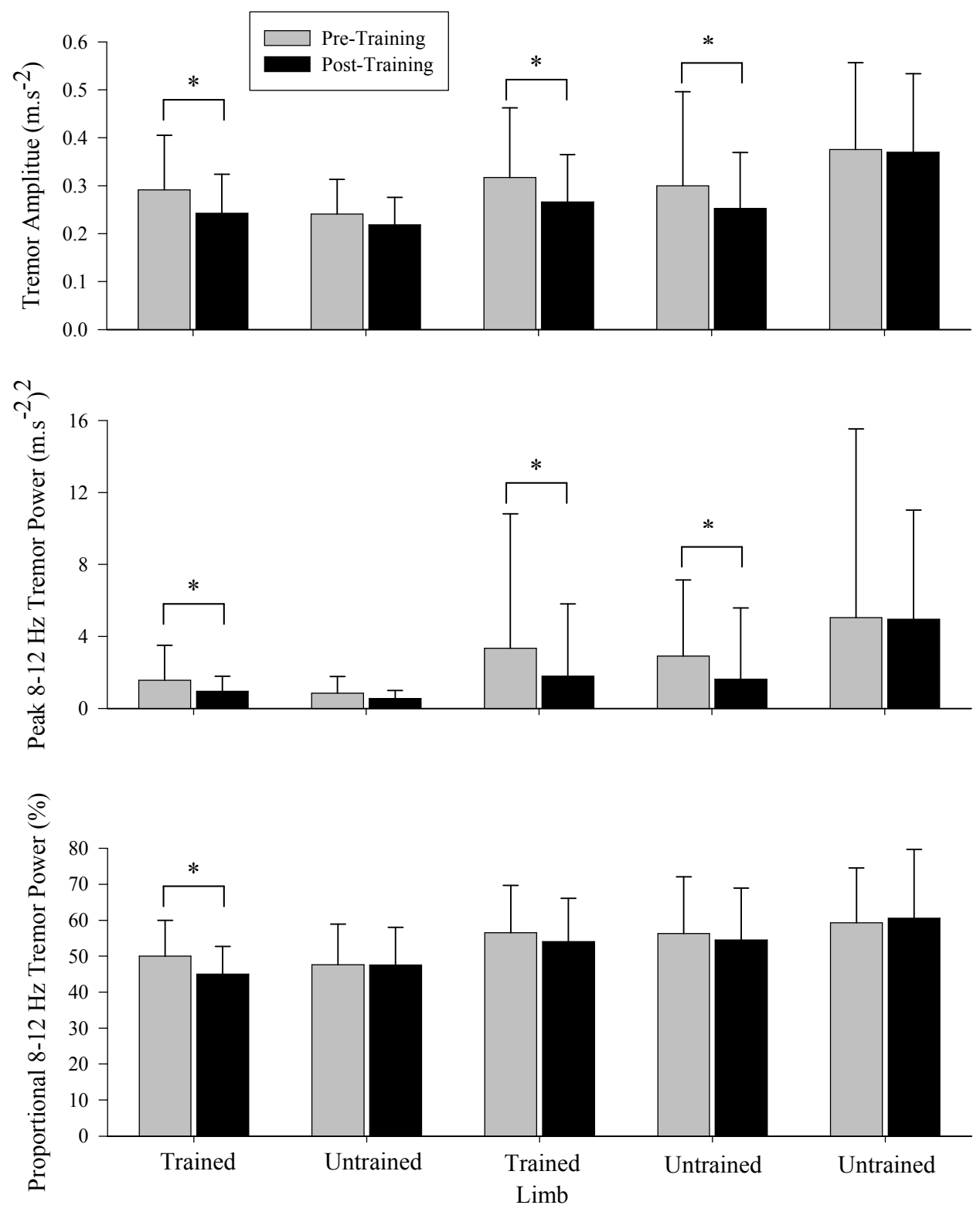

Figure 5 - Pre- and posttraining tremor amplitude and 8- to $12-\mathrm{Hz}$ peak and proportional power scores for the strength- and coordination-training and control groups, $M \pm S D$. * Significantly greater change for the training versus control group. 


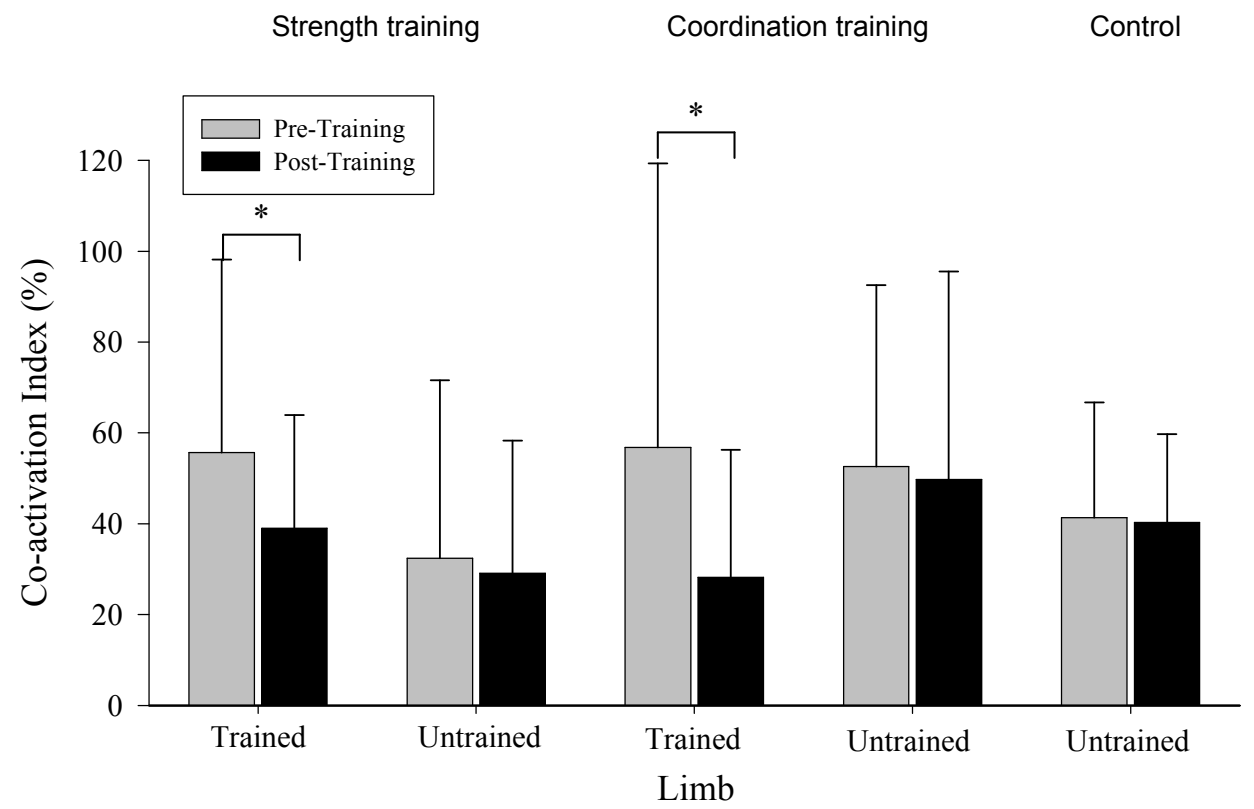

Figure 6 - Pre- and posttraining coactivation index (CI) scores for the strength- and coordinationtraining and control groups, $M \pm S D$. *Significantly greater change for the training versus control group.

This result was inconsistent with our initial hypothesis, whereby it was predicted that the greater coordinative challenge afforded by the semispecific coordinationtraining tasks would produce a larger reduction in tremor amplitude than conventional strength training. Although both forms of resistance training elicited similar reductions in the tremor amplitude of the older adults, the lack of statistical power for such a comparison may have contributed to this result. Further research in this area should use randomized controlled trial models involving larger sample sizes and training durations if the aim is to more clearly demonstrate whether coordinationtraining protocols have greater potential than strength training to reduce the tremor amplitude of older adults.

To date, only two other studies have examined the chronic effect of resistance training on the tremor output of older adults (Bilodeau et al., 2000; Ranganathan, Siemionow, Saugen, et al., 2001). Similar to our results, Ranganathan, Siemionow, Saugen, et al. observed significant improvements in neurologically healthy older adults' hand steadiness (an indirect measure of tremor amplitude) in a precise finger-pinch task. This improvement in hand steadiness was observed after 8 weeks of dexterity-ball training that involved a variety of dexterous finger-pinching movements. In contrast, Bilodeau et al. reported no significant change in the tremor amplitude of middle-aged to older essential-tremor patients after 4 weeks of isometric index-finger-abduction training. Bilodeau et al. found that such training was able to significantly reduce index-finger-abduction force variability. Collectively, 
the findings of the current study and Ranganathan, Siemionow, Saugen, et al. demonstrate that there is some potential for exercise interventions to reduce postural tremor amplitude in neurologically healthy older adults. Although previous findings suggest that resistance training may only reduce older adults' tremor amplitude in tasks similar to those performed in training (Bilodeau et al.; Ranganathan, Siemionow, Saugen et al.), the current study demonstrated this effect in actions that shared no similarities with the training exercises. It therefore appears that as long as the resistance-training exercises engage the relevant agonist-antagonist muscles involved in the transfer tasks, the exercise prescription need not be overly specific. The generality of this response is of much practical benefit because older adults would not have sufficient time to practice all the tasks in which they might experience an age-related decline in ability.

We hypothesized that significant cross-education effects (i.e., improvements in the function of the untrained limb) would also occur as a consequence of the training interventions. The results demonstrated that the contralateral (untrained) limb experienced significant increases in wrist-flexion strength (strength-training group) and significant reductions in tremor amplitude (coordination-training group). Moderate to large $(E S>.50)$ but nonsignificant cross-education effects were also observed for biceps-curl and wrist-extension strength in the strength-training group and biceps-curl and wrist-flexion strength in the coordination-training group.

Although several reviews indicate that young adults can obtain significant cross-education strength benefits from unilateral resistance training (Lee \& Carroll, 2007; Zhou, 2000), only two studies have shown this effect in older adults (Bemben \& Murphy, 2001; Tracy et al., 1999). Our results support the results of these two studies, indicating that older adults are able to increase the strength of the untrained limb after unilateral resistance training. Of further interest is the coordination-training group's significant cross-education tremor-amplitude effect. Currently, the literature on cross-education indicates that the untrained limb can experience significant improvements in muscle strength as long as the testing task is identical or similar to that performed in training by the trained limb (Lee \& Carroll; Zhou). The results of the current study extend this perspective by demonstrating that older adults are able to obtain significant cross-education effects in tasks requiring dexterity, as well as strength tests, and not performed in training. This result is of practical importance because it suggests that unilateral resistance training may be able to play a role in the rehabilitation of patients with unilateral neurological or orthopedic conditions (Lee \& Carroll). Future research should determine the clinical significance of the cross-education effects and the optimal loading patterns for various patient groups.

We expected that any reductions in tremor amplitude would be most pronounced between 8 and $12 \mathrm{~Hz}$ and would also be associated with a decrease in upper limb muscle coactivation. Our results supported this view, demonstrating that the strength- and coordination-training groups experienced significant reductions in peak 8 - to $12-\mathrm{Hz}$ tremor power and upper limb muscle coactivation but no change in the tremor within other frequency ranges $(2-4 \mathrm{~Hz}$ and $18-24 \mathrm{~Hz}$ ). Because the 8- to $12-\mathrm{Hz}$ tremor peak is derived from a number of central and peripheral neural inputs (McAuley \& Marsden, 2000) and because significant reductions in coactivation were observed for the training groups, it is possible that neural adaptations were responsible for the reduced activity of some of the upper limb muscles and 
ultimately the 8 - to $12-\mathrm{Hz}$ component of index-finger tremor. It is also possible that these changes merely reflected the increases in upper limb strength. Regardless of the mechanism responsible for these changes, our results share some similarities with two studies involving pathological-tremor patients (Chung, Poppen, \& Lundervold, 1995; Lundervold, Belwood, Craney, \& Poppen, 1999). These two studies reported that biobehavioral (biofeedback) training decreased upper limb muscle activity or coactivation and tremor output in these patients. It is therefore possible that any intervention that reduces upper limb muscle activity or coactivation may also reduce the tremor output of neurologically healthy older adults, as well as those with pathological tremor.

A limitation of the current study is the lack of random allocation of participants to each of the three groups. As a result, the strength-training group had significantly less wrist-flexion and -extension strength, as well as tremor amplitude, than the control group at baseline. Participants with low baseline strength levels (i.e., the strength-training group) are likely to experience larger increases in strength in 6 weeks than stronger individuals, with much of this increase in strength reflecting neural adaptations to training (Barry \& Carson, 2004; Hakkinen et al., 1998). These weaker individuals are therefore more likely to obtain considerable trainingrelated neural adaptations that may allow them to reduce their tremor amplitude. Alternatively, participants with low baseline tremor amplitude (i.e., the strengthtraining group) may have less potential to reduce their tremor than those with higher tremor amplitude regardless of the task or intervention performed (Keogh et al., 2004). Because the baseline differences in strength and tremor output for the strength-training and control groups act in opposite directions with respect to the magnitude of the expected training-related change in tremor amplitude, they may have tended to negate each other. However, it is possible that the benefit of resistance training of reducing postural tremor in older adults is greatest for individuals with reduced upper limb strength or mildly enhanced tremor. Regardless of the design limitations (e.g., random allocation of participants, different baseline characteristics of the strength training and control group), the lack of any significant baseline differences between the coordination-training and control groups would indicate that the coordination-training group's reduction in tremor amplitude was likely to be a true result.

\section{Conclusions}

The major finding of the current study was that two resistance-training-based exercise interventions significantly reduced the tremor amplitude of neurologically healthy older adults. After strength or coordination training, all participants exhibited an increase in upper limb strength, a reduction in upper limb muscle coactivation, and subsequently a reduction in 8- to $12-\mathrm{Hz}$ postural tremor amplitude. Because there were considerable differences between the testing (transfer) and training tasks, it appears that older adults can significantly reduce postural tremor amplitude as a result of performing resisted gross motor tasks involving moderately sized muscle groups. These findings further support the prescription of resistance training for older adults and suggest that the reduction in tremor amplitude was primarily a result of neural adaptations to training. 


\section{References}

Barry, B.K., \& Carson, R.G. (2004). The consequence of resistance training for movement control in older adults. The Journals of Gerontology. Series A, Biological Sciences and Medical Sciences, 59(7), 530-554.

Bemben, M.G., \& Murphy, R.E. (2001). Age related neural adaptation following short term resistance training in women. Journal of Sports Medicine and Physical Fitness, 41, 291-299.

Bilodeau, M., Keen, D.A., Sweeney, P.J., Shields, R.W., \& Enoka, R.M. (2000). Strength training can improve steadiness in persons with essential tremor. Muscle \& Nerve, 23, 771-778.

Birmingham, A.T., Wharrad, H.J., \& Williams, E.J. (1985). The variation of finger tremor with age in man. Journal of Neurology, Neurosurgery, and Psychiatry, 48, 788-798.

Brunner, F., Schmid, A., Sheikhzadeh, A., Nordin, M., Yoon, J., \& Frankel, V. (2007). Effects of aging on Type II muscle fibers: A systematic review of the literature. Journal of Aging and Physical Activity, 15(3), 336-348.

Carmeli, E., Patish, H., \& Coleman, R. (2003). The aging hand. The Journals of Gerontology. Series A, Biological Sciences and Medical Sciences, 58(2), M146-M152.

Carolan, B., \& Cafarelli, E. (1992). Adaptations in coactivation after isometric training. Journal of Applied Physiology, 73(3), 911-917.

Chung, W., Poppen, R., \& Lundervold, D.A. (1995). Behavioral relaxation training for tremor disorders in older adults. Biofeedback and Self-Regulation, 20, 123-135.

Cohen, J. (1988). Statistical power analysis for the behavioral sciences. Hillsdale, NJ: Lawrence Erlbaum.

A Collaborative Outcomes Research Network. (2007). Pre-post change score. Retrieved October 20, 2008, from www.psychoutcomes.org/bin/view/OutcomesMeasurement/ ChangeScore

DeLuca, C.J. (1997). The use of surface electromyography in biomechanics. Journal of Applied Biomechanics, 13, 135-161.

Enoka, R.M., Christou, E.A., Hunter, S.K., Kornatz, K.W., Semmler, J.G., Taylor, A.M., et al. (2003). Mechanisms that contribute to differences in motor performance between young and old adults. Journal of Electromyography and Kinesiology, 13, 1-12.

Fiatarone-Singh, M.A. (2002). Exercise comes of age: Rationale and recommendations for a geriatric exercise prescription. The Journals of Gerontology. Series A, Biological Sciences and Medical Sciences, 57A(5), M262-M282.

Hackel, M.E., Wolfe, G.A., Bang, S.M., \& Canfield, J.S. (1992). Changes in hand function in the aging adult as determined by Jebsen Test of Hand Function. Physical Therapy, 72, 373-377.

Hakkinen, K., Kallinen, M., Izquierdo, M., Jokelainen, K., Lassila, H., Malkia, E., et al. (1998). Changes in agonist-antagonist EMG, muscle CSA, and force during strength training in middle-aged and older people. Journal of Applied Physiology, 84(4), 1341-1349.

Hopkins, W.G. (2002). A new view on statistics: Log transformation. Retrieved March 5, 2005, from http://sportsci.org/resource/stats/index.html\#Log.

Hortobagyi, T., \& DeVita, P. (2000). Muscle pre- and coactivity during downward stepping are associated with leg stiffness in aging. Journal of Electromyography and Kinesiology, 10, 117-126.

Hrysomallis, C., \& Kidgell, D. (2001). Effect of heavy dynamic resistive exercise on acute upper body power. Journal of Strength and Conditioning Research, 15(4), 426-430.

Keogh, J., Morrison, S., \& Barrett, R. (2004). Augmented visual feedback increases finger tremor during postural pointing. Experimental Brain Research, 159, 467-477.

Keogh, J.W., Morrison, S., \& Barrett, R. (2007). Strength-training improves the tri-digit finger-pinch force control of older adults. Archives of Physical Medicine and Rehabilitation, 88, 1055-1063. 
Knight, C.A., \& Kamen, G. (2004). Enhanced motor unit rate coding with improvements in a force-matching task. Journal of Electromyography and Kinesiology, 14(6), 619-629.

Kornatz, K.W., Christou, E.A., \& Enoka, R.M. (2005). Practice reduces motor unit discharge variability in a hand muscle and improves manual dexterity in old adults. Journal of Applied Physiology, 98(6), 2072-2080.

Kosek, D.J., Kim, J-s., Petrella, J.K., Cross, J.M., \& Bamman, M.M. (2006). Efficacy of 3 days/wk resistance training on myofiber hypertrophy and myogenic mechanisms in young vs. older adults. Journal of Applied Physiology, 101(2), 531-544.

Laidlaw, D.H., Bilodeau, M., \& Enoka, R.M. (2000). Steadiness is reduced and motor unit discharge is more variable in old adults. Muscle \& Nerve, 23, 600-612.

Laursen, B., Jensen, B.R., \& Ratkevicius, A. (2001). Performance and muscle activity during computer mouse tasks in young and elderly adults. European Journal of Applied Physiology, 84, 329-336. Lee, M., \& Carroll, T.J. (2007). Cross education: Possible mechanisms for the contralateral effects of unilateral resistance training. Sports Medicine (Auckland, N.Z.), 37(1), 1-14.

Loscher, W.N., \& Gallasch, E. (1993). Myoelectric signs of muscle fatigue and physiological tremor from childhood to seniority. In G.E. Stelmach \& V. Homberg (Eds.), Sensorimotor impairment in the elderly (pp. 103-127). Dordrecht, The Netherlands: Kluwer Academic.

Lundervold, D.A., Belwood, M.F., Craney, J.L., \& Poppen, R. (1999). Reduction of tremor severity and disability following behavioural relaxation training. Journal of Behavior Therapy and Experimental Psychiatry, 30, 119-135.

McAuley, J.H., \& Marsden, C.D. (2000). Physiological and pathological tremors and rhythmic central motor control. Brain, 123, 1545-1567.

Morrison, S., \& Keogh, J. (2001). Changes in the dynamics of tremor during goal-directed pointing. Human Movement Science, 20, 675-693.

Morrison, S., Mills, P., \& Barrett, R. (2006). Differences in multiple segment tremor dynamics between young and elderly persons. The Journals of Gerontology. Series A, Biological Sciences and Medical Sciences, 61(9), 982-990.

Moss, B.M., Refsnes, P.E., Abildgaard, A., Nicolaysen, K., \& Jensen, J. (1997). Effects of maximal effort strength training with different loads on dynamic strength, crosssectional area, load-power and load-velocity relationships. European Journal of Applied Physiology, 75(3), 193-199.

Patten, C., \& Kamen, G. (2000). Adaptations in motor unit discharge activity with force control training in young and older human adults. European Journal of Applied Physiology, 83, 128-143.

Ranganathan, V.K., Siemionow, V., Saghal, V., \& Yue, G. (2001). Effects of aging on hand function. Journal of the American Geriatrics Society, 49, 1478-1484.

Ranganathan, V.K., Siemionow, V., Saugen, E., Liu, J.Z., \& Yue, G.H. (2001). Skilled finger movement improves hand function. The Journals of Gerontology. Series A, Biological Sciences and Medical Sciences, 56(8), M518-M522.

Sosnoff, J.J., \& Newell, K.M. (2006). Are age-related increases in force variability due to decrements in strength? Experimental Brain Research, 174(1), 86-94.

Spiegel, K.M., Stratton, J., Burke, J.R., Glendinning, D.S., \& Enoka, R.M. (1996). The influence of age on the assessment of motor unit activation in a human hand muscle. Experimental Physiology, 81(5), 805-819.

Tracy, B.L., Ivey, F.M., Hurlbut, D., Martel, G.F., Lemmer, J.T., Siegel, E.L., et al. (1999). Muscle quality. II. Effects of strength training in 65- to 75-yr-old men and women. Journal of Applied Physiology, 86(1), 195-201.

Zhou, S. (2000). Chronic neural adaptations to unilateral exercise: Mechanisms of cross education. Exercise and Sport Sciences Reviews, 28(4), 177-184. 